\title{
VISIBILITY BASED CONTINUUM SUBTRACTION
}

\author{
HUIB JAN VAN LANGEVELDE
}

Sterrewacht Leiden, Postbus 9513, 2300 RA LEIDEN, The Netherlands

\section{BILL COTTON}

N.R.A.O. Charlottesville, Edgemont Road, Charlottesville, VA 22901, U.S.A.

ABSTRACT We have been looking into the possibility to subtract out the continuum contribution in spectral line databases from the ungridded visibilities. It turns out that our simple method offers quite interesting possibilities in a lot of practical cases. In this contribution we show how and why this method works. Details of this work can be found in van Langevelde and Cotton (1990). We have tried to compare the results of this method with the more conventional ways of subtracting the continuum. This comparison has to be done with simulations of which we show some results.

\section{INTRODUCTION}

When doing a spectral line experiment one usually likes to examine the maps of the spectral line image with the continuum removed. This can be a very time consuming process, especially when a simple subtraction of maps does not produce satisfying results.

Our motivation to look in to other possibilities to subtract the continuum was a project to monitor the variability of $\mathrm{OH} / \mathrm{IR}$ stars in the Galactic center. In these observations $\mathrm{SgrA}$ is a source of bright extended emission (see figure 1 ), which has to be removed to measure the flux of the $\mathrm{OH}$ masers accurately. The big advantage of visibility based subtraction in this case is that it uses little CPU-time and disk space; important here, since we have to reduce \pm 20 observations of a field with 30 masers.

\section{HOW DOES IT WORK?}

We have implemented a simple algorithm in an AIPS task called UVBAS. The principle of this task is very straightforward. Two ranges in frequency are specified which are assumed to be free of line emission. These will be used to estimate the continuum contribution in the line channels. Because UV-data come from the interferometer as "visibility spectra", we have the visibilities from a baseline at a certain time across the spectrum. In this spectrum we 
make a weighted average of the "empty" channels and subtract that from the channels of interest.

Even if individual "visibility spectra" are dominated by noise, this will subtract out the continuum, because these linear combinations correspond to linear operations in the map plane. This is different from the approach to fit the phases and amplitudes in the visibilities (see Uson, Bagri and Cornwell, these proceedings). The latter method is in principle better suited to deal with structure in the "visibility spectra" (phase winds) across the band. It will therefore work better in larger bandwidth cases and in fields dominated by point sources. To do this however it requires reasonable $\mathrm{S} / \mathrm{N}$ in individual visibilities. Here we concentrate on the algorithm which we use for our low $S / N$ data.
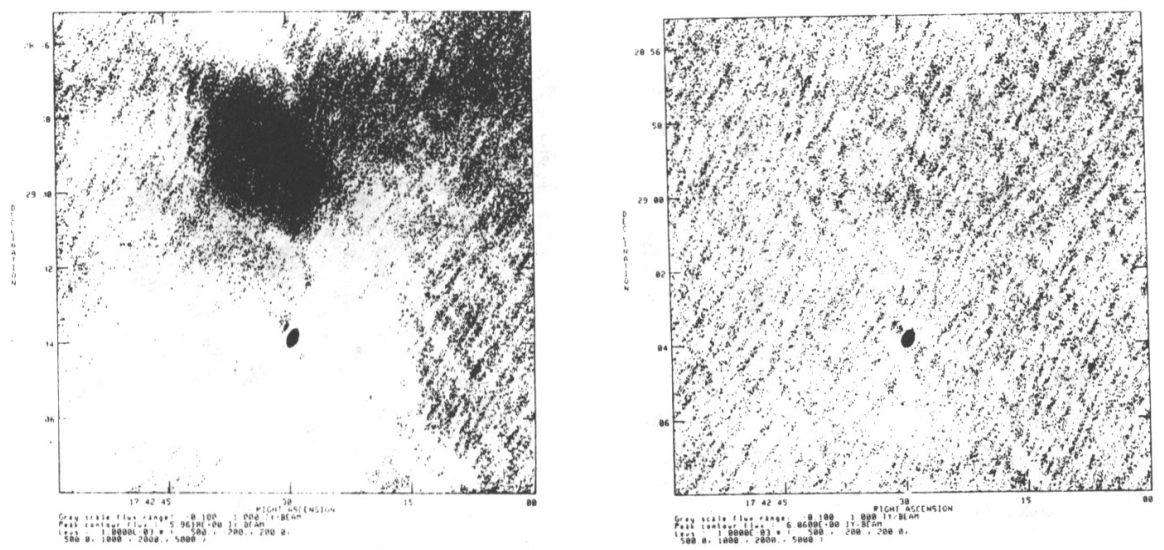

Fig. 1. a. A map of one channel of OH359.88-0.087 near SgrA, observed with the VLA in $\mathrm{C}$ array, channelwidth is $6.3 \mathrm{kHz}$. b. Same as a, but now after UVBAS, we used two ranges of 10 channels, 38 channels apart to subtract the continuum.

The limits of our method

Clearly our subtraction method introduces errors, because the same baseline at a different frequency is in fact sampling a different place in the UV-plane. Part of this effect is compensated by making a linear interpolation across the "visibility spectrum". But a second order effect remains that scales with baseline length, relative bandwidth over which we try this trick and the speed with which the visibilities change from one place to another in the UV-plane. It will thus introduce a systematic residual in the map. If this residual is sufficiently low compared to the noise level, the method is useful.

\section{COMPARISON}

It is obviously important to know how well "visibility based subtraction" works in comparison with other schemes to subtract the continuum. The errors we introduce with UVBAS can be estimated, for other methods that turns out to be difficult. We have performed a number of simulations on several artificial 
datasets. These datasets were noise free and we compared the methods by the highest residuals left after subtraction. All pictures in this paper were produced from the same database, it resembles the VLA in C array at 1612 $\mathrm{Mhz}$, and we try to subtract continuum emission over $\approx 200 \mathrm{kHz}$.

\section{Compared with image subtraction}

When subtracting images, residual structure originates in the differences in beam with frequency. UVBAS overcomes this limit, but introduces errors of a different nature. In different simulations and in several tests on real data however we have never encountered a case where image subtraction reaches more satisfying results. UVBAS is, including mapping, something like 30 times faster.
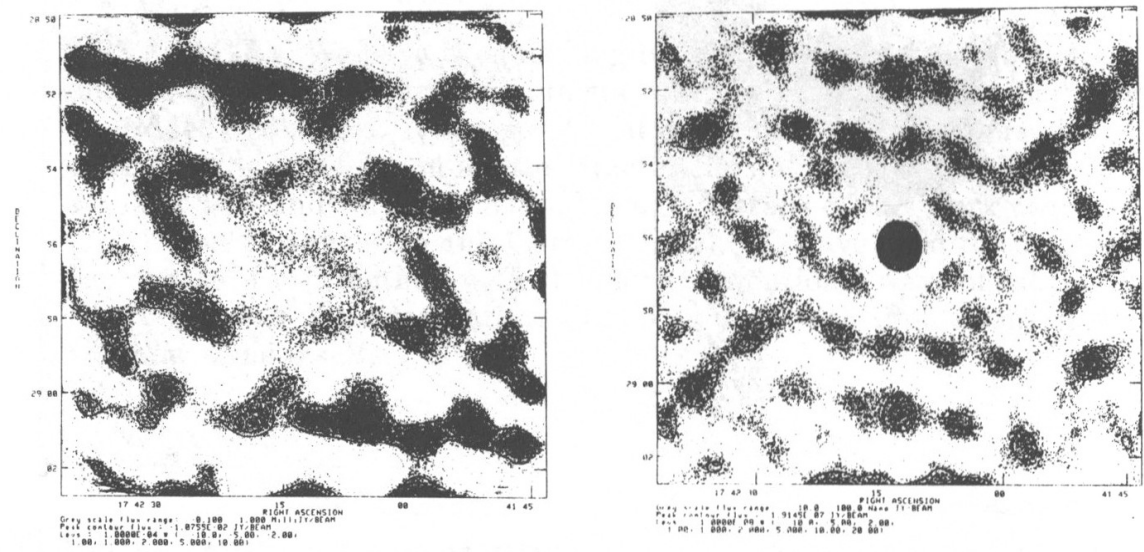

Fig. 2. Simulation with an artificial dataset with a $100 \mathrm{Jy}$ gaussian. A noisefree dataset was created with the same "observing parameters" as in figure 1. a. The residual image after the dirty image of the gaussian was subtracted out in the image plane; we averaged images at different frequencies and subtracted this model from the original. Maximum residual: $3 \mathrm{mJy}$. b. The residual image after the image with the gaussian was treated by UVBAS, notice the totally different scale of this figure, maximum residual is $0.2 \mu \mathrm{Jy}$.

\section{Compared with UVSUB}

In this scheme one forms a model of the continuum, for example by CLEANing, next the fourier transform of this model is subtracted from the visibility data (van Gorkom and Ekers, 1989). The mentioned different respons of the interferometer at different frequecies is also corrected for in this way. So in principle this method is always correct, and should be able to work better than UVBAS, especially in cases of broad bandwidth. In simulations with small bandwidth however, we have noticed some cases were the intrinsic (numerical) limits of CLEAN in fact set some ultimate level which can be reached. In these cases UVBAS seems to work better. We should mention though that this level will never be reached in practical observations due to limited bandpass stability. A more important consideration here is the 
difference in computer time consumption. UVBAS takes about 400 times less CPU-time.

\section{CONCLUSIONS}

We conclude that visibility based subtraction can be usefull in specific cases. We think that it can be used instead of image based continuum subtraction in all cases. It also pushes the limit where one is forced to remove the continuum by subtracting a model image from the UV-data, especially in cases of small bandwidth.

The main reason why one would prefer a visibility based subtraction scheme when possible is its low consumption of computer resources.
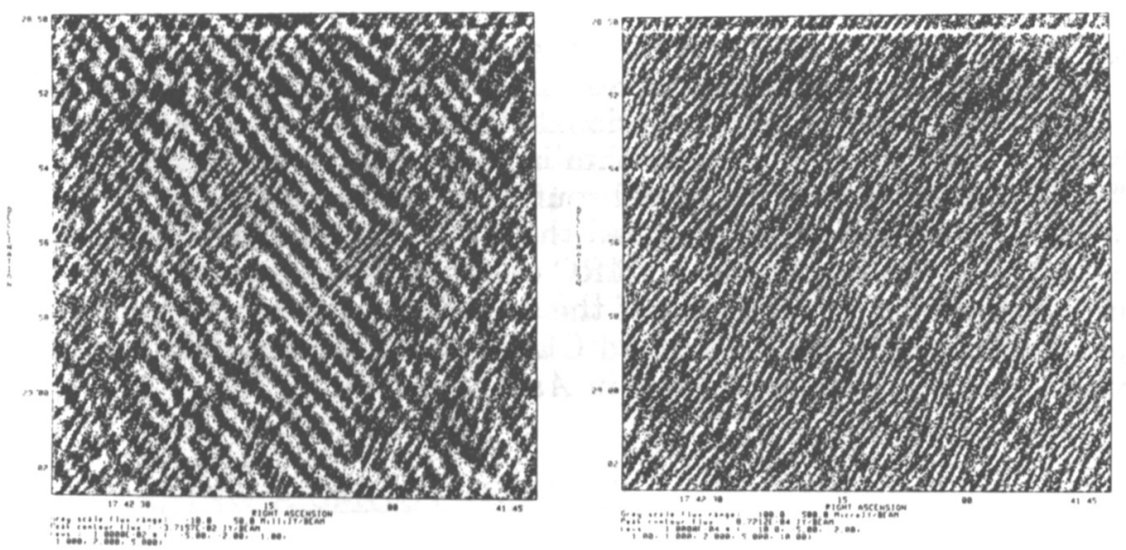

Fig. 2. Simulations with 5 100Jy point sources. Same "observing parameters" as above. b The residual map after we CLEANed an average map of bracketing frequencies and subtracted the list of clean componenents from the visibility data. Maximum residual: $40 \mathrm{mJy}$. c. The residual image after the image with 5 point source was treated by UVBAS. Levels in figure are different, maximum residual is $700 \mu \mathrm{Jy}$.

\section{ACKNOWLEDGEMENTS}

This work greatly benefitted from discussions with Juan Uson, Jacqueline van Gorkom and Tim Cornwell. H.J.v.L acknowledges partial travel support from NATO grant No. 870545.

\section{REFERENCES}

van Gorkom J.H., Ekers R.D., 1989, in Synthesis imaging in radio astronomy, eds. Perley, Schwab and Bridle (Astronomical Society of the Pacific, San Francisco) p 341

van Langevelde, H. J., Cotton, W.D., 1990, Astr. Ap. Lett., in press 